\title{
Incompleteness of Standard Model regarding Einstein's Program
}

\author{
Claude Elbaz \\ Correspondence: Claude Elbaz, Academie Europeenne Interdisciplinaire de Science (A.E.I.S.), Paris, France. \\ Email: claude.elbaz@science-inter.com
}

Received: January 20, 2018

doi:10.11114/set.v5i1.3446

\author{
Accepted: February 28, 2018 \\ Online Published: July 16, 2018 \\ URL: https://doi.org/10.11114/set.v5i1.3446
}

\begin{abstract}
The Standard Model of Particles gathers almost the whole present knowledge in theoretical physics, in a consistent system. It describes the universe as constituted fundamentally of particles, both for matter and for interactions. It is incomplete since it does not include gravitation with the three other interactions, and leans upon elementary particles such as electrons or quarks, which are considered as point-like with no known internal structure. The Einstein's program offers means to circumvent these difficulties. It is founded upon a scalar field $\varepsilon\left(\mathrm{r}_{0}, \mathrm{t}_{0}\right)$ propagating at speed of light $\mathrm{c}$, as a consistent system for universe description. Standing waves correspond to matter, which kinematical and dynamical properties are formally identical. Their space-like amplitude function $\mathrm{u}_{0}\left(\mathrm{k}_{0} \mathrm{r}_{0}\right)$, supplements usual fundamental time-like equations of classical and quantum mechanics. In geometrical optics approximation conditions, when frequencies are infinitely high, and then hidden, $\mathrm{u}_{0}\left(\mathrm{k}_{0} \mathrm{r}_{0}\right)$ yields Dirac's distribution $\delta\left(\mathrm{r}_{0}\right)$, describing a particle as a singularity. The scalar field $\varepsilon\left(\mathrm{r}_{0}, \mathrm{t}_{0}\right)$ brings theoretical economy by yielding also the energy-momentum conservation laws, and the least action law, with determination of the Lagrangian.The frequency variations of the field $\varepsilon\left(\mathrm{r}_{0}, \mathrm{t}_{0}\right)$ are linked to electromagnetic interaction, constituted by progressive waves. Quantum domain corresponds to wave optics approximation conditions, with wave-particle duality. Adiabatic variations of frequencies, give rise to introduction of an adiabatic constant, formally identical with Planck's constant, with unification of first quantification for interactions and second quantification for matter. Local variations of speed of light yield gravitation interaction and General Relativity framework.
\end{abstract}

Keywords: Einstein's program, Standard Model, Quantum Field Theory, adiabatic invariant, wave-particle duality

\section{Introduction}

The experimental detection of gravitational waves represents the last, and most recent, important discovery in physics. The first direct detection, largely reported by the mass media, occurred in 2016, one century after the prediction by Einstein, following equations of General Relativity. As a mark of importance for the scientific community, the Nobel's prize was promptly attributed to the contributors in 2017.Then, after one century of efforts, carried on by different physicists in the world, Einstein's works reappeared again at the front of research in physics.

Such a renewed scientific visibility of Einstein at the front of research, after a long lapse of time, occurred beyond, and despite, the prodigious successful development of the probabilistic approach of the quantum theory, to which he remained constantly, and resolutely opposed. We notice that the equations of General Relativity, he established before, lean on a classical and determinist framework. Thus, in one sense, the experimental detection of gravitational waves reinforces the Einstein's reliance upon determinism in physics.

This is not without raising many questions, especially if we notice that just only few years ago, in 2012, the detection ofthe Higgs boson, was previously considered as the most important discovery in physics. As the crowning of quantum theory, it represented the experimental validation of the probabilistic behavior of universe through the Standard Model of particles. It was largely reported by the mass media, and was already followed by a prompt attribution of the Nobel's prize, to the contributors in 2013.

Thus, after more than one half century of efforts, in a very short lapse of time of few years, the two important alternative currents of theoretical physics, were separately validated, and reinforced, by experiment: the determinist and classical current following General Relativity, and the probablistic and quantum current following Quantum Mechanics.

Until gravitational waves were directly detected, the probabilistic quantum theory, was most largely admitted by physicists, owing to the numerous successful results upon theory, experiment, and associated technology. It led to the 
Standard Model of Particles, which gathers almost the whole present knowledge in theoretical physics. It describes the universe in a consistent system, as constituted fundamentally of particles, both for matter and for interactions.

However, a glimpse at the table of constitutive Elementary Particles (Wikipedia, 2017) shows that the Standard Model is incomplete, since two domains are at least missing: gravitation, beside the three other present interactions, (strong, weak and electromagnetic), and size of particles, beside the three other present characteristics, (mass, charge and spin).

\section{Standard Model of Elementary Particles}

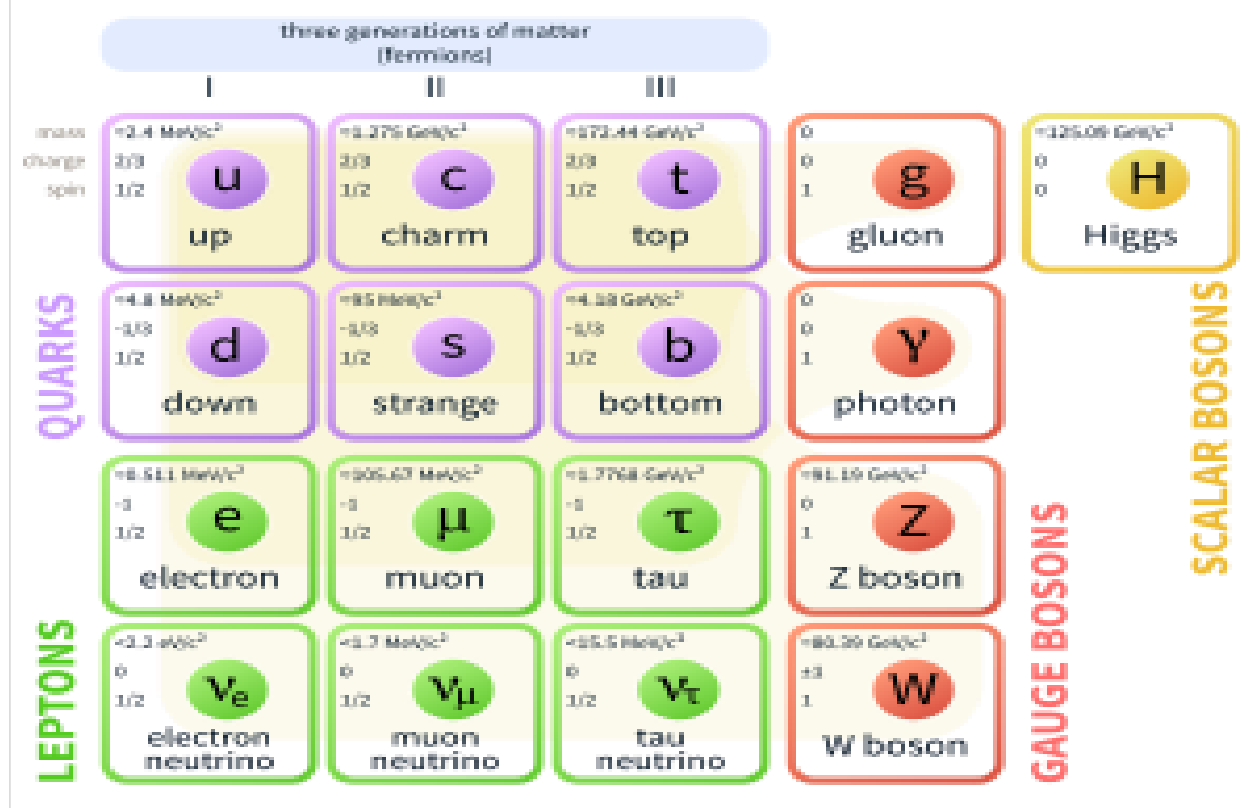

It is admitted that both missing domains are not so important in order to have an effect upon the contents of the Standard Model. For gravitation, its weakness, as compared with other interactions, makes that it does not play a significant rôle at particles level. For the size of individual particles, it is not considered of prime importance, since investigations bear principally upon the determination of their energy rather than their position, which are directly linked to their size.

The absence of gravitation, experimentally justified, explains particularly why the Standard Model ignores General Relativity: the Special Relativity framework remains largely sufficient. It yields also the material particles to be considered as point-like, with zero dimension, as singularities of the field (Landau, 1962) (Einstein, 1938). The experimental detection of gravitational waves, predicted independently before the introduction of probablist Quantum Mechanics, points out that the gravitation stays out the framework of the Standard Model. In one hand, gravitation, as interaction, still remains described by classical and deterministic equations of General Relativity of Einstein, with an improved great precision. In another hand, therecent experiment detected waves, and not particles, as expected gravitons.

The Einstein's reappearance at the front of research, through gravitational waves, incites to actualize his resolute opposition to the probabilistic approach of Quantum Mechanics, beyond the above incompleteness of the Standard Model which extends it. Then after one century, the Einstein's statement holds unchanged.

Despite his reliance upon determinism in universe, Einstein recognized that the probabilistic framework of the Quantum Mechanics was experimentally successful and theoretically coherent, even though its mathematical formalism appeared quite amazing. "This double nature of radiation (and of material corpuscles) is a major property of reality, which has been interpreted by quantum mechanics in an ingenious and amazingly successful fashion. This interpretation, which is looked upon as essentially final by almost all contemporary physicists, appears to me as only a temporary way out." (Einstein, 1949)

For Einstein, the probabilistic experimental behaviour of quantum particles, like electrons, proves only that the quantum mechanics description is incomplete. «The statistical character of the present theory would then have to be a necessary consequence of the incompleteness of the description of the systems in quantum mechanics...Above all, however, the reader should be convinced that I fully recognize the very important progress which the statistical quantum theory has brought in physics.... this theory is until now the only one which unites the corpuscular and undulatory dual character 
of matter in a logically satisfactory fashion; and the (testable) relations, which are contained in it, are, within the natural limits fixed by the undeterminacy-relation, complete. The formal relations which are given in this theory-i,e, its entire mathematical formalism - will probably have to be maintained, in the form of logical inferences, in every useful future theory." (Einstein, 1938)

At this stage, it is worthwhile to emphasize that the Einstein's contributions upon statistics in physics are essential. For instance, in the standard Model, one class of elementary particles, the bosons, are so designated because they obey the Bose-Einstein's quantum law of statistics. In addition, he introduced the statistical theory treatment of the Brownian motion in 1905, and discovered the quantum stimulated emission of light in atoms In 1916 (Einstein, 1916) by prolonging the treatment of the Brownian motion (Dalibard, 2016).

Consequently, he expected that, in the future, Quantum Mechanics, and by extension present Standard Model, will be completed and wholly integrated as an approximation, without any internal modifications, inside a more general framework. We can compare such a process with two preceding examples, resulting from Einstein's own works: the Newton's laws of mechanics inside those of Special Relativity, and the Newton's laws of celestial mechanics inside those of General Relativity. In both cases, the Mechanics of Newton was incorporated as a whole, without any internal modifications, in a more general framework, as approximations. Nevertheless, the previous Newton's laws still remain usefully utilized, and largely sufficient, in numerous usual technical applications.

It seems worthwhile to point out some scientific and personal reasons which justify why Einstein was founded to disagree with the probabilistic orientation of quantum theory, and to privilege a classical deterministic conception of physics.

From a scientific point of view, not only he brought essential contributions to the deterministic current with Special Relativity in 1905 followed by General Relativity in 1916, but each time, almost simultaneously, he carried out essential contributions to the quantum theory, together with statistics. In 1905, he introduced the first quantum particle with energy light quanta $E=h v$, afterward designated as photon, from the photoelectric effect and the statistical theory treatment of the Brownian motion. In 1916, he discovered the quantum stimulated emission of light in atoms (Einstein, 1916) by prolonging the treatment of the Brownian motion (Dalibard, 2016).

We can appreciate the importance of Einstein's contribution in quantum theory, by noticing that the Nobel's prize was awarded to him in 1921 not for classical Relativity theories, but for his introduction of the quantum particle, which behaves as a particle and as a wave. We notice that, nowadays, the elementary quantum particles form the core of the Standard Model. In years 1905 and 1916 Einstein's important publications show that the classical and the quantum frameworks, together with statistical behaviors, where not dissociated in his mind.

Since the 1920 years,after the wave-particle duality problems had much mobilized physicists, the probabilistic and mathematical abstract framework was selected to build Quantum Mechanics.

From a personal point of view, Einstein was dissatisfied by such an orientation. He was accustomed to give priority to physical models for representations, and to rely upon thoughts experiments, like for some famous controversies with Bohr. We find such physical models for representations, and thoughts physical experiments, at the basis of his theories of Relativity: with the reciprocal electrodynamic action of a magnet and a conductor, at origin of Special Relativity, and with the motion of bodies in free fall,

for General Relativity. For him:«Most of the fundamental ideas of science are essentially simple, and may, as a rule, be expressed in a language comprehensible to everyone. To follow up these ideas demands the knowledge of a highly refined technique of investigation. Mathematics as a tool of reasoning is necessary if we wish to draw conclusions which may be compared with experiment. So long as we are concerned only with fundamental physical ideas, we may avoid the language of mathematics (Einstein, 1938)

Nevertheless the Einstein's dissatisfaction was not limited to Quantum Mechanics, but concerned also General Relativity. He thought it was incomplete, because of its material basis constitued by point-like particles, or singularities. "One would be compelled to demand that the particles themselves would everywhere be described as singularity-free solutions of the completed field-equations. Only then would the general theory of relativity be a complete theory." (Einstein, 1949).

His different works convinced him that the extended field, rather than the particle, would be privileged in order to investigate the basic constitution of universe (Einstein, 1920, 1929, 1936). In his Program, the field would yield a more general framework, not only for the Standard Model, but also for General Relativity. «We have two realities: matter and field. ....We cannot build physics on the basis of the matter concept alone. But the division into matter and field is, after the recognition of the equivalence of mass and energy, something artificial and not clearly defined. Could we not reject the concept of matter and build a pure field physics? ...We could regard matter as the regions in space where the field is extremely strong. In this way a new philosophical background could be created.... Only field-energy would be left, and the particle would be merely an area of special density of field-energy. In that case one could hope to deduce the concept of the mass-point together with the equations of the motion of the particles from the field equations- the disturbing dualism would have been 
removed... One would be compelled to demand that the particles themselves would everywhere be describable as singularity free solutions of the completed field-equations. Only then would the general theory of general relativity be a complete theory.... One could believe that it would be possible to find a new and secure foundation for all physics upon the path which had been so successfully begun by Faraday and Maxwell.» (Einstein, 1938).

From an experimental point of view, since the 1960 years, the Einstein's Program has been implicitly validated and implemented by the International Legal Metrology Organization, when, after been used during two centuries, matter was replaced by field, in order to determine the standard for measures of length and time. Nowadays they are based on one particular period of an electromagnetic wave frequency (Dimarcq, 2013). In addition, the velocity of light in vacuum is admitted as a primary fundamental constant in physics, with its numerical value strictly fixed. By comparison, the Planck's constant is not yet considered as a primary fundamental constant for determination of the standard for measures of mass. Such a difference of statute credits Einstein's attitude to consider the velocity of light and the determinism as more fundamental than the Planck's constant and the probabilism.

From a theoretical point of view, owing to the prominent role of experiment and measures in physics, we are led to banish rigid rods and particles, as well as material clocks, in basic space-time representations of matter, on behalf of waves propagating at light velocity.

From a technological point of view, the Einstein's Program, even implicitly, is more generally and progressively implemented, when we notice that all usual material devices are, almost systematically, replaced by numerical and electromagnetic ones.

In this article, we propose to show how the Einstein's Program offers a way to complete the Standard Model of Particles. According to it, "it would be possible to find a new and secure foundation for all physics upon the path which had been so successfully begun by Faraday and Maxwell.»Starting from a scalar field $\varepsilon(\mathrm{r}, \mathrm{t})$ propagating at speed of light, we get a consistent system for universe description, at the same time for the Standard Model of Particles, and for General Relativity. It completes them by deriving a point-like material particle as an approximation limit of the field, instead of admitting it as a fundamental singularity, in order to fit with experiment. The kinematical and dynamical properties of standing waves, which can be in motion with a speed strictly inferior to speed of light, correspond to those of matter. In addition, the scalar field offers theoretical economies by yielding the energy-momentum conservation law, the least action principle with determination of the Lagrangian, the electromagnetic interaction from local variations of waves frequencies, and the gravitation interaction from local variations oflight speed, instead of admitting them as fundamental laws or principles. Classical domain corresponds to geometrical optics approximations, when very high frequencies are undetectable and hidden. Quantum domain corresponds to wave optics conditions. Since a field cannot physically extend to infinity, the necessarily limiting homogeneous boundaries result from local variations of waves frequencies, yielding Fourier relations, formally identical with Heisenberg relations. Very small variations of frequencies, give rise to an adiabatic invariant constant, formally identical with Planck's constant, yielding unification of first quantification for interactions and second quantification for matter, and to wave-particle duality properties.

\section{The Einstein's Program}

We restrict to summarize some equations deduced from Einstein's program, previously published, (Elbaz, 2010, 2012, 2013, 2014), in order to show how they are related to main equations of classical and quantum mechanics, otherwise widely documented.

\subsection{Kinematical Properties of Standing Fields}

Starting from the d'Alembertian's equation describing a scalar field $\varepsilon$ propagating at constant light velocity c, we make all following results sure that they hold in the special relativist framework

$$
\square \varepsilon=\Delta \varepsilon-\left(1 / \mathrm{c}^{2}\right)\left(\partial^{2} \varepsilon / \partial \mathrm{t}^{2}\right)=0, \quad \quad \partial^{\mu} \partial_{\mu} \varepsilon=0
$$

It yields two kinds of elementary harmonic solutions with constant frequency $\omega_{0}$, with different kinematical properties: progressive and standing waves. Oscillating progressive waves, either retarded like $\cos \left(\omega_{0} \mathrm{t}_{0}-\mathrm{k}_{0} \mathrm{x}_{0}\right)$ or advanced like $\cos \left(\omega_{0} \mathrm{t}_{0}+\mathrm{k}_{0} \mathrm{x}_{0}\right)$, are in motion at light velocity $\mathrm{c}=\omega_{0} / \mathrm{k}_{0}$. Standing waves, where space and time variables are separated, like $\varepsilon_{0}\left(\mathrm{x}_{0}, \mathrm{t}_{0}\right)=\mathrm{u}_{0}\left(\mathrm{k}_{0} \mathrm{x}_{0}\right) \psi_{0}\left(\omega_{0} \mathrm{t}_{0}\right)=\cos \left(\omega_{0} \mathrm{t}_{0}\right) \cos \left(\mathrm{k}_{0} \mathrm{x}_{0}\right)$, oscillate locally. They allow to define a system of coordinates at rest $\left(\mathrm{x}_{0}, \mathrm{t}_{0}\right)$.

They may be considered as resulting from superposition of two progressive waves

$$
\cos \left(\omega_{0} \mathrm{t}_{0}+\mathrm{k}_{0} \mathrm{x}_{0}\right)+\cos \left(\omega_{0} \mathrm{t}_{0}-\mathrm{k}_{0} \mathrm{x}_{0}\right)=2 \cos \left(\omega_{0} \mathrm{t}_{0}\right) \cos \left(\mathrm{k}_{0} \mathrm{x}_{0}\right) .
$$

When, in a system of reference $(\mathrm{x}, \mathrm{t})$, the frequencies of opposite progressive waves are different

$$
\cos \left(\omega_{1} t-k_{1} x\right)+\cos \left(\omega_{2} t+k_{2} x\right)=2 \cos (\omega t-\beta k x) \cos (k x-\beta \omega t),
$$

where $\omega=\left(\omega_{1+} \omega_{2}\right) / 2=k c$, and $\beta=\left(\omega_{1}-\omega_{2} / \omega_{1+} \omega_{2}\right)$. By identification with (2), they form a standing wave in motion with a 
speed $v=\beta c=\left(\omega_{1}-\omega_{2} / \omega_{1+} \omega_{2}\right) c$, Its frequency $\omega=\left(\omega_{1+} \omega_{2}\right) / 2=k c$ corresponds to a rest frequency $\omega_{0}=\sqrt{ } \omega_{1} \omega_{2}$, defining the Lorentz transformation between the systems of reference at rest $\left(\mathrm{x}_{0}, \mathrm{t}_{0}\right)$ and in motion $(\mathrm{x}, \mathrm{t})$, and leading to its whole physical relativist consequences.

It can be shown that the Lorentz transformation, fundamental in special relativity, is specific of c-field standing waves, particularly through the coefficient $\sqrt{ }\left(1-\beta^{2}\right)$. which becomes $(1 \pm \beta)$ for progressive waves (Elbaz,2014). The four-dimensional Minkowski's formalism expresses invariance properties of standing waves at rest, in which the variables of space and time are separated, when they move uniformly with a speed $\mathrm{v}$, necessarily inferior to $\mathrm{c}$, since $\beta$ is a relative difference $v=\beta c<c$. Confirmation is found into invariant quantities obtained from four-quantities, such as coordinates $\mathrm{x}_{\mu} \mathrm{x}^{\mu}=\mathrm{x}_{0}^{2}$ or $\mathrm{x}_{\mu} \mathrm{x}^{\mu}=\mathrm{c}^{2} \mathrm{t}_{0}^{2}$, and functions $\mathrm{u}_{\mu} \mathrm{u}^{\mu}=\mathrm{u}^{2}\left(\mathrm{x}_{0}\right)$ or $\psi_{\mu} \psi^{\mu}=\psi^{2}\left(\mathrm{t}_{0}\right)$. Their space-like or time-like characters are absolute, depending of their refering coordinates defined in the rest system.

Since the functions $\mathrm{u}_{0}\left(\mathrm{k}_{0} \mathrm{x}_{0}\right)$ and $\psi_{0}\left(\omega_{0} \mathrm{t}_{0}\right)$ are independent, the frequency $\omega_{0}$ is necessarily constant in

$$
\left(1 / \mathrm{u}_{0}\right) \Delta_{0} \mathrm{u}_{0}=\left(1 / \psi_{0}\right)\left(\partial^{2} \psi_{0} / \mathrm{c}^{2} \partial \mathrm{t}_{0}{ }^{2}\right)=-\mathrm{k}_{0}{ }^{2}=-\omega_{0}{ }^{2} / \mathrm{c}^{2} .
$$

The function of space $\mathrm{u}_{0}\left(\mathrm{k}_{0} \mathrm{x}_{0}\right)$, obeys the Helmholtz's equation at rest $\Delta_{0} \mathrm{u}_{0}+\mathrm{k}_{0}{ }^{2} \mathrm{u}_{0}=0$, becomes in moving system $(\mathrm{x}, \mathrm{t})$, $\Delta \mathrm{u}-\left(1 / \mathrm{c}^{2}\right)\left(\partial^{2} \mathrm{u} / \partial \mathrm{t}^{2}\right)+\mathrm{k}_{0}{ }^{2} \mathrm{u}=0$. It describesgeometrical properties of standing waves. It verifies Bessel spherical functions solutions, and particularly its simplest elementary solution, with spherical symmetry, finite at origin of the reference $\operatorname{system}\left(\mathrm{x}_{0}, \mathrm{t}_{0}\right)$

$$
\mathrm{u}_{0}\left(\mathrm{k}_{0} \mathrm{r}_{0}\right)=\left(\sin \mathrm{k}_{0} \mathrm{r}_{0}\right) /\left(\mathrm{k}_{0} \mathrm{r}_{0}\right) .
$$

It leads to Dirac's distribution $\mathrm{u}_{0}\left(\mathrm{k}_{0} \mathrm{r}_{0}\right) \rightarrow \delta\left(\mathrm{r}_{0}\right)$ when frequency tends towards infinity $\omega_{0}=\mathrm{k}_{0} \mathrm{c} \rightarrow \propto$, in Cartesian system of reference, the central extremum of an extended standing wave determines its position $\mathrm{x}_{0}=\mathrm{r}_{0}=0$.

$$
\nabla_{0} u_{0}\left(\mathbf{x}_{0}\right)=0 .
$$

In order to point out the constant frequency of a moving standing field, we express it as

$$
\varepsilon(\omega t, k x)=u(k x, \beta c t) \exp i(\omega t-\beta k x) \quad \varphi=\omega t-\beta k x
$$

The equations of special and general relativity are based on mass-points, as singularities, moving on trajectories, deriving then directly from geometrical optics approximation. Consequently, for a scalar field propagating at light velocity $\mathrm{c}$, with constant frequency wand constant velocity $\mathrm{v}$, the kinematic properties of standing waves, reduce formally to kinematical properties of isolated point-like matter.

\subsection{Dynamical Properties of Standing Fields}

Since a field $\varepsilon(\omega t, \mathrm{kx})$ cannot extend physically to infinity with respect to space and time, one imposes usually boundary conditions exerted by matter in order to limit it. Matter behaves either as a source, which fixes the wavelength $\lambda$ through $\mathrm{k}=2 \pi / \lambda$ and then the frequency $\omega=\mathrm{kc}$, or as a detector annealing it. In addition to its heterogeneous character, this is not felicitous from relativistic consistency, since space and time operate separately.

In order to stay in a homogeneous frame, we consider two progressive waves with different frequencies $\omega_{1}, \omega_{2}$ propagating in the same direction at light velocity. They give rise to a wave packet propagating in the same direction at light velocity. Its main wave with frequency $\omega=\left(\omega_{1+} \omega_{2}\right) / 2$, is modulated by a wave with frequency $\beta \omega=\left(\omega_{1}-\omega_{2}\right) / 2=\Delta \omega / 2=\Delta \mathrm{kc} / 2$, wavelength $\Lambda=2 \pi / \beta \mathrm{k}$, and period $\mathrm{T}=\Lambda / \mathrm{c}$. Since $\beta<1$, The modulation wave acts as an envelope with space and time extensions $\Delta \mathrm{x}=\Lambda / 2, \Delta \mathrm{t}=\mathrm{T} / 2$, leading to well known Fourier relations $\Delta \mathrm{x} . \Delta \mathrm{k}=2 \pi$ and $\Delta \mathrm{t} . \Delta \omega=2 \pi$.

The boundary conditions for the scalar field $\varepsilon(\omega t, \mathrm{kx})$ are represented by the Fourier relations, which must supplement d'Alembertian's equation (1), in order to emphasize that the field cannot extend to infinity with respect to space and time. When the frequencies difference $\beta \omega=\left(\omega_{1}-\omega_{2}\right) / 2=\Delta \omega / 2<<\omega$ is very small, it can be considered as a perturbation with respect to the main frequency, $\beta \omega=\delta \omega$.

An almost monochromatic wave can be characterized by a frequency $\Omega(\mathrm{x}, \mathrm{t})$, almost constant $\omega$,

$$
\Omega(\mathrm{x}, \mathrm{t})=\mathrm{K}(\mathrm{x}, \mathrm{t}) \mathrm{c}=\omega \pm \delta \Omega(\mathrm{x}, \mathrm{t}) \quad \delta \Omega(\mathrm{x}, \mathrm{t})<<\omega \quad \omega=\text { constant } .
$$

We retrieve the definition of an adiabatic variation for the frequency (Ehrenfest, 1911). Consequently, all following properties of almost fields arise inside such a process. In that framework, the necessarily constant frequency of a standing wave must be considered as the mean value, all over the field, of different slowly varying frequencies $\Omega(x, t)$, instead of a given data. The perturbation frequencies of modulation waves propagating at light velocity $\delta \Omega(\mathrm{x}, \mathrm{t})$, behave then as interactions between main waves, yielding the mean frequency $\omega$ to remain practically constant all over the space-time (Elbaz, 2012).

Such a behavior authorizes mathematically to derive the properties of almost fields with varying frequency $\Omega(x, t)$ from monochromatic ones with constant mean frequency $\omega$, through the variation of constants method (Duhamel principle). 
Following (8), we express it, as

$$
\varepsilon(\mathrm{x}, \mathrm{t})=\mathrm{U}(\mathrm{x}, \mathrm{t}) \exp \mathrm{i} \phi(\mathrm{x}, \mathrm{t}) \quad \phi(\mathrm{x}, \mathrm{t})=\Omega(\mathrm{x}, \mathrm{t}) \mathrm{t}-\mathbf{K}(\mathrm{x}, \mathrm{t}) \cdot \mathrm{x}+2 \mathrm{n} \pi,
$$

where products of second order $\delta \Omega d t \approx 0$ and $\delta \mathbf{K} . \mathrm{d} \mathbf{x} \approx 0$,, defined modulo $2 \pi$, are neglected at first order of approximation. This is equivalent to incorporate directly the boundary conditions defined by Fourier relations, in almost monochromatic solutions,

$$
\mathrm{d} \phi(\mathrm{x}, \mathrm{t})=\Omega(\mathrm{x}, \mathrm{t}) \mathrm{dt}-\mathbf{K}(\mathrm{x}, \mathrm{t}) . \mathrm{d} \mathbf{x} \approx \omega \mathrm{dt}-\mathrm{k} . \mathrm{d} \mathbf{x} . \quad \mathrm{U}(\mathrm{x}, \mathrm{t})=\mathrm{u}(\mathrm{x}, \mathrm{t}) \pm \delta \mathrm{U}(\mathrm{x}, \mathrm{t})
$$

According to (1), the field $\varepsilon(\omega t, k x)$ defined by (9) verifies,

$$
\begin{aligned}
\partial^{\mu} \partial_{\mu} \mathrm{U}-\mathrm{U} \partial^{\mu} \phi \partial_{\mu} \phi=0 & \text { or } \quad \partial^{2} \mathrm{U} / \mathrm{c}^{2} \partial \mathrm{t}^{2}-\nabla^{2} \mathrm{U}-\mathrm{U}\left[(\partial \phi / \mathrm{c} \partial \mathrm{t})^{2}-(\nabla \phi)^{2}\right]=0 \\
\partial^{\mu}\left(\mathrm{U}^{2} \partial_{\mu} \phi\right)=0 & \text { or } \quad \partial\left(\mathrm{U}^{2} \Omega\right) / \mathrm{c}^{2} \partial \mathrm{t}+\nabla \cdot\left(\mathrm{U}^{2} \beta \mathbf{K}\right)=0
\end{aligned}
$$

These relations apply to progressive waves for $\beta= \pm 1$, to standing waves at rest for $\beta=0$ and in motion for $\beta<1$, to monochromatic waves for $\omega$ and $\mathbf{k}$ constant, to almost monochromatic waves for varying $\Omega(\mathrm{x}, \mathrm{t})$ and $\mathbf{K}(\mathrm{x}, \mathrm{t})$. They lead to dynamical properties for energy-momentum conservation, and to least action, principles, for standing fields and for almost standing fields ( Elbaz, 1984, 2010, 2014, 2015).

For a standing wave with constant frequency $\omega$, either at rest or in motion, (12) reduces to

$$
\partial \mathrm{u}_{0}{ }^{2} / \partial \mathrm{t}_{0}=0 . \quad \partial \mathrm{u}^{2} / \partial \mathrm{t}+\nabla \cdot \mathrm{u}^{2} \mathbf{v}=0 \quad \text { or } \quad \partial_{\mu} \mathrm{w}^{\mu}=0
$$

Where $\mathrm{w}^{\mu}=\left(\mathrm{u}^{2}, \mathrm{u}^{2} \mathbf{v} / \mathrm{c}\right)=\mathrm{u}_{0}\left(\mathrm{x}_{0}\right)^{2}(1, \mathbf{v} / \mathrm{c}) / \sqrt{ }\left(1-\beta^{2}\right)$ is a four-dimensional vector. This continuity equation for $\mathrm{u}^{2}$ is formally identical with the Newton's equation continuity for matter-momentum density $\partial \mu / \partial t+\nabla \cdot \mu \mathbf{v}=0$. By transposition, we can then admit that $\mathrm{u}^{2}$ represents the energy density of the standing field.

The centre of amplitude, with position $\mathrm{x}_{0}$ defined by (6), as a characteristic point of a standing field, allows to determine its kinematical behavior. Then, the position $\mathrm{x}_{0}$ of the energy density verifies

$$
\nabla_{0} \mathbf{u}_{0}^{2}=0 \quad \nabla \mathrm{u}^{2}+\left(\partial \mathrm{u}^{2} \mathbf{v} / \mathrm{c}^{2} \partial \mathrm{t}\right)=0 \nabla \times \mathbf{v}=0 \quad \text { or } \quad \pi^{\mu v}=\partial^{\mu} \mathrm{w}^{v}-\partial^{v} \mathrm{w}^{\mu}=0,
$$

The standing wave energy density $\mathrm{u}^{2}$, which is as spread in space, corresponds to a potential energy density. Then, $\mathbf{F}=$ $-\nabla u^{2}=-\nabla w_{P}$ is a density force, and $\partial u^{2} v / c^{2} \partial t$ is a density momentum. They are gathered in $\pi^{\mu v}$ as a four-dimensional force density.

By identification of density momentum $u^{2} \mathbf{v} / \mathrm{c}^{2}$ of field and $\mu \mathbf{v}$ of matter, we retrieve the relation of equivalence of equivalence between mass and energy

$$
\mathrm{u}^{2} \mathrm{v} / \mathrm{c}^{2}=\mu \mathrm{v} \quad \text { or } \quad \mathrm{u}^{2}=\mu \mathrm{c}^{2} .
$$

We notice that the light velocity c did not appear in equation of continuity for energy density (13), because it is constant, in the present special relativist framework, like in the Standard Model. We will show farther that this is no longer true in the general relativity framework of gravitation.

In equation (14), the vanishing four-dimensional force density tensor $\pi^{\mu \nu}$ of a standing wave, asserts that its space stability at rest holds in motion, and that the energy-momentum density four-vector $\mathrm{w}^{\mu}$ is four-parallel, or directed along the motion velocity $\mathbf{v}$.

Equation (14) is mathematically equivalent to the least action principle, in which energy density $w^{\mu}$ is a four-dimensional gradient $\partial^{\mu} \mathrm{a}$,

$$
\delta \int \mathrm{da}=0 \quad \delta \int \partial^{\mu} \operatorname{adx}_{\mu}=0 \quad \text { with } \quad \mathrm{w}^{\mu}=\partial^{\mu} \mathrm{a} .
$$

When we transpose the mass density $\mu=\mathrm{u}^{2} / \mathrm{c}^{2}$, and we take into account the identities $\nabla \mathrm{P}^{2}=2(\mathbf{P} . \nabla) \mathbf{P}+2 \mathbf{P} \times(\nabla \times \mathbf{P})$ and $\mathrm{d} \mathbf{P} / \mathrm{dt}=\partial \mathbf{P} / \partial \mathrm{t}+(\mathbf{v} . \nabla) \mathbf{P}$ forc and $\mathrm{v}$ constant, after integration with respect to space, we obtain from (14) the equation for matter

$$
\mathrm{d} / \mathrm{dt}=-\nabla \mathrm{mc} \mathrm{c}^{2}+\left\{\nabla(\mathrm{mv})^{2}\right\} / 2 \mathrm{~m} \quad \mathrm{~d} \mathbf{p} / \mathrm{dt}=\nabla \mathrm{L}_{\mathrm{m}}=-\nabla \mathrm{m}_{0} \mathrm{c}^{2} \sqrt{ }\left(1-\beta^{2}\right) .
$$

We retrieve the relativistic Lagrangian of mechanics for free matter $L_{m}=-m_{0} c^{2} \sqrt{ }\left(1-\beta^{2}\right)$.

Then kinematical and dynamical properties of standing fields are identical with those of free matter.

\subsection{Electromagnetic Interaction}

For an almost standing wave, the continuity equation (12), relates to the total energy density, $\quad \mathrm{W}=\mathrm{U}^{2} \Omega=\mathrm{W}+\delta \mathrm{W}$, sum of the mean energy density $\mathrm{w}$ of the standing wave with high constant frequency $\omega$, and of the interactions $\delta \mathrm{W}$ with lower varying frequency $\delta \Omega(\mathrm{x}, \mathrm{t})$. The relation (14) becomes

$$
\Pi^{\mu v}=\partial^{\mu} \mathrm{W}^{v}-\partial^{v} \mathrm{~W}^{\mu}=0 \quad \text { or } \quad \Pi^{\mu v}=\pi^{\mu \nu}+\delta \Pi^{\mu v}=0
$$

The total density force $\Pi^{\mu v}$ for an almost standing wave vanishes. This traduces its stability when in motion. Its total 
energy-momentum density four-vector $\mathrm{W}^{\mu}$ is directed along the constant moving velocity $\mathrm{v}$.

By difference with the null four-dimensional density force $\pi^{\mu v}$ for a standing wave, only the total density force $\Pi^{\mu v}$ for an almost standing wave vanishes. In the first case, this asserts the space stability of free, or isolated, moving standing wave, while in the second case, the space stability concerns the whole almost standing wave. It behaves as a system composed of two sub-systems, the mean bounded standing field with almost constant high frequency $\Omega(\mathrm{x}, \mathrm{t}) \approx \omega$, , and the interaction field with lower frequency $\delta \Omega(\mathrm{x}, \mathrm{t})$. Each ones exerts an equal and opposite density force $\pi^{\mu v}=-\delta \Pi^{\mu v}$ against the other. For the mean bounded standing wave, the energy-momentum density tensor $\pi^{\mu v}$ no longer vanishes in (18), as previously in (14). This comes from the mean energy-momentum density four-vector $w^{\mu}$, which is no longer parallel, because of the opposite density force $\delta \Pi^{\mu v}$ exerted by the interaction.

It appears that an almost standing field behaves as a whole system in motion, which can be split in two sub-systems, the mean standing field and the interaction field. Both are moving with velocity $\mathrm{v}$, while exerting each other opposite equal forces in different directions, including perpendicularly to the velocity v. The perturbation field, arises from local frequency variations $\delta \Omega(\mathrm{x}, \mathrm{t})$, behaving as interaction. It introduces orthogonal components in interaction density force and momentum.

The relations (17) generalized by constants variation method $M(x, t)=m \pm \delta M(x, t)$, becomes

$$
\nabla \mathrm{Mc}^{2}+\partial \mathrm{P} / \partial \mathrm{t}=0 \quad \nabla \times \mathrm{P}=0 \quad \mathrm{dP} / \mathrm{dt}=-\nabla \mathrm{Mc}^{2}+\left(\nabla \mathrm{P}^{2}\right) / 2 \mathrm{M} .
$$

The non vanishing density force $\delta \Pi^{\mu v} \neq 0$ exerted by the interaction, is formally identical with the electromagnetic tensor $\mathrm{F}^{\mu v}=\partial^{\mu} \mathrm{A}^{v}-\partial^{v} \mathrm{~A}^{\mu} \neq 0$. We can set them in correspondence $\delta \Pi^{\mu v}=\mathrm{eF}^{\mu v}$, through a constant invariant charge $\mathrm{e}$, with $\delta \mathrm{M}(\mathrm{x}, \mathrm{t})=$ $\mathrm{eV}(\mathrm{x}, \mathrm{t}) / \mathrm{c}^{2}$ and $\delta \mathrm{P}(\mathrm{x}, \mathrm{t})=\mathrm{eA}(\mathrm{x}, \mathrm{t}) / \mathrm{c}$. The double sign for the mass variation corresponds to the two signs for electric charges, or to emission and absorption of electromagnetic energy by matter. We retrieve the minimum coupling of classical electrodynamics, $\mathrm{P}^{\mu}(\mathrm{x}, \mathrm{t})=\mathrm{p}^{\mu}+\mathrm{eA}^{\mu}(\mathrm{x}, \mathrm{t}) / \mathrm{c}$, with $\mathrm{M}(\mathrm{x}, \mathrm{t}) \mathrm{c}^{2}=\mathrm{mc}^{2}+\mathrm{eV}(\mathrm{x}, \mathrm{t})$, and $\mathrm{P}(\mathrm{x}, \mathrm{t})=\mathrm{p}+\mathrm{eA}(\mathrm{x}, \mathrm{t}) / \mathrm{c}$, where electromagnetic energy exchanged with a particle is very small compared to its own energy $\mathrm{eA}^{\mu}(\mathrm{x}, \mathrm{t}) / \mathrm{c}=\delta \mathrm{P}^{\mu}(\mathrm{x}, \mathrm{t})<<$ $\mathrm{p}^{\mu}$ (Landau,1962). The electromagnetic interaction is then directly linked to frequencies variations of the field $\varepsilon(\omega t, k x)$.

Relation (19) yields the relativistic Newton's equation for charged matter with the Lorentz force

\subsection{Adiabatic Invariant}

$$
\mathrm{dP} / \mathrm{dt}=-\nabla \mathrm{m}_{0} \mathrm{c}^{2} \sqrt{ }\left(1-\beta^{2}\right)+\mathrm{e}(\mathrm{E}+\mathrm{v} \times \mathrm{H} / \mathrm{c})
$$

For an almost standing wave, the relation (11) leads to first order approximation to

$$
\left[\partial \mathrm{U}^{2} / \partial \mathrm{t}+\nabla \cdot \mathrm{U}^{2} \mathbf{v}\right] / \mathrm{U}^{2}+\delta[\partial \Omega / \partial \mathrm{t}+\nabla \cdot \Omega \mathbf{v}] / \Omega=0 \quad \text { or } \quad\left(\partial_{v} \mathrm{~W}^{v}\right) / \mathrm{W}+\delta\left(\partial_{v} \Omega^{v}\right) / \Omega=0
$$

where $\mathrm{W}=\mathrm{W} \pm \delta \mathrm{W}=\mu \mathrm{c}^{2}=\mu \mathrm{c}^{2} \pm \delta \mu \mathrm{c}^{2}$ is its total energy density, and $\mu$ the corresponding mass density, $\mathrm{W}^{v}=\mathrm{w}^{v} \pm \delta \mathrm{W}^{v}$, the four-dimensional energy density, $\Omega=\omega \pm \delta \Omega$, the frequency, and $\Omega^{v}=(\Omega, \Omega v / c)$, the four-dimensional frequency, leading to

$$
\mathrm{W}^{v}=\mathrm{I} \Omega^{v} \text { or } \quad \mathrm{W}^{v}=\mathrm{I} \omega^{v} \quad \text { and } \delta \mathrm{W}^{v}=\mathrm{I} \delta \Omega^{v}
$$

when we take into account the double sign in frequency variation $\delta \Omega$. The constant I is an adiabatic invariant density. It is the same constant which links the energy-momentum density $\mathrm{W}^{v}$ of the almost standing wave, with the four-dimensional frequency $\delta \Omega^{v}$ of the interaction, their respective small variations $\delta \mathrm{W}^{v}$ and $\delta \Omega^{v}$.

After integrations with respect to space, the adiabatic invariant density I becomes adiabatic invariant $H$. Four-dimensional energy densities $W^{v}$ and $\delta W^{v}$ become four-dimensional energy $E^{v}$ and $\delta E^{v}$. We get the relations, between energy and momentum of the mean field of an almost monochromatic standing field

and its small variations,

$$
\mathrm{E}^{v}=\mathrm{H} \Omega^{v} \text { or } \quad \mathrm{E}=\mathrm{mc}^{2}=\mathrm{H} \omega \quad \mathbf{p}=\mathrm{mv}=\mathrm{Hk}
$$

$$
\mathrm{dE}^{v}=\mathrm{Hd} \Omega^{v} \quad \text { or } \quad \mathrm{dE}=\mathrm{c}^{2} \mathrm{dm}=\mathrm{Hd} \Omega \mathrm{d} \mathbf{p}=\mathbf{v} \mathrm{dm}=\mathrm{Hd} \mathbf{k}
$$

which correspond to either absorbed, or emitted, energy and momentum of interactions

$$
\left.\mathrm{dE}^{v}=\mathrm{d} \mathrm{E}^{\mathrm{v}}(\mathrm{x}, \mathrm{t}) \quad \text { or } \quad \mathrm{dE}=\mathrm{d} \delta \mathrm{E}(\mathrm{x}, \mathrm{t}) \quad \mathrm{d} \mathbf{p}=\mathrm{d} \delta \mathrm{P}(\mathrm{x}, \mathrm{t})\right)
$$

They are formally equivalent with quantum relations for energy-momentum of a material particle and its interactions, with the adiabatic invariant $\mathrm{H}$ corresponding to the reduced Planck's constant $\mathrm{h} / 2 \pi$.

If the standing field $\varepsilon_{0}\left(\mathrm{x}_{0}, \mathrm{t}_{0}\right)=\mathrm{u}_{0}\left(\mathrm{k}_{0} \mathrm{x}_{0}\right) \psi_{0}\left(\omega_{0} \mathrm{t}_{0}\right)$ in (4), relates to an electron, the adiabatic invariant $\mathrm{H}$ becomes identical with the Planck's constant $\mathrm{h}$, yielding the relations (23-25) as the de Broglie-Einstein's relations $\mathrm{E}=\mathrm{mc}^{2}=\mathrm{h} \omega / 2 \pi$ at the basis of Wave Mechanics.

For the standing wave corresponding to matter, local adiabatic variations of its frequency $\Omega(\mathrm{x}, \mathrm{t})$ lead to electromagnetic interaction constituted by progressive waves (Elbaz, 2012). The energy of electromagnetic interaction derives from 
mass variation $d E=c^{2} d m$. It leans directly upon wave properties of matter, in which its energy $d E=h d v=c^{2} d m$ derives from variations of matter energy.

\subsection{Gravitation and General Relativity}

All above results derived from a scalar field $\varepsilon$ propagating with a constant light velocity $\mathrm{c}$ in vacuum, according to the d'Alembertian equation (4). The standing waves properties, characterized in a rest system $\left(\mathrm{x}_{0}, \mathrm{t}_{0}\right)$ by separated space and time variables $\varepsilon_{0}\left(\mathrm{x}_{0}, \mathrm{t}_{0}\right)=\mathrm{u}_{0}\left(\mathrm{k}_{0} \mathrm{x}_{0}\right) \psi_{0}\left(\omega_{0} \mathrm{t}_{0}\right)$, and a constant frequency $\omega_{0}=\mathrm{k}_{0} \mathrm{c}$, hold their space stability when they are in uniform motion. This is expressed by the arising Lorentz transformation.

Before its application to mechanics, and more generally to special relativistic physics, the Lorentz transformation characterized the structure of Maxwell's equations in vacuum. The presence of matter, as support of electric charges, modifies locally the field properties, through induced dielectric and magnetic permeability variations, so that the field velocity propagation $\mathrm{c}$ is no longer constant, and must be written $\mathrm{C}(\mathrm{x}, \mathrm{t})$ (Elbaz, 2015). For instance, In optics, the variations are linked to a refraction index $n(x, t)=c / C(x, t)$, characteristic ofthe medium, which is no longer empty.

Since almost standing waves behave like localized matter, we may expect that, as a secondary effect, their energy density $\mathrm{u}^{2}=\mu \mathrm{c}^{2}$ modifies very slightly the light velocity propagation of the field, so that it remains close to its vacuum constant value, becoming $\mathrm{C}(\mathrm{x}, \mathrm{t})=\mathrm{c} \pm \delta \mathrm{C}(\mathrm{x}, \mathrm{t})$, with $\delta \mathrm{C}(\mathrm{x}, \mathrm{t})<<\mathrm{c}$. The relation $\mathrm{v}=\beta \mathrm{c}$ shows that the local variations of the motion velocity v must follow, leading to $\mathrm{V}(\mathrm{x}, \mathrm{t})=\mathrm{v} \pm \delta \mathrm{V}(\mathrm{x}, \mathrm{t})$, with $\delta \mathrm{V}(\mathrm{x}, \mathrm{t})<<\mathrm{v}$. Consequently, the Lorentz transformation, in which the velocities $\mathrm{v}$ and $\mathrm{c}$ are constant, appears only as expressing a local approximation limit, from a more general form with varying velocities, yielding slight variations of frequency $\Omega(\mathrm{x}, \mathrm{t})=\mathrm{K}(\mathrm{x}, \mathrm{t}) \mathrm{C}(\mathrm{x}, \mathrm{t})$, and wave vector $\mathrm{K}(\mathrm{x}, \mathrm{t})=\mathrm{k} \pm \delta \mathrm{K}(\mathrm{x}, \mathrm{t})$.Then, in a rest system, space and time terms are no longer fully separated in expression $\varepsilon_{0}\left(\mathrm{x}_{0}, \mathrm{t}_{0}\right)=\mathrm{U}_{0}\left(\mathrm{x}_{0}, \mathrm{t}_{0}\right) \Psi_{0}\left(\mathrm{x}_{0}, \mathrm{t}_{0}\right)$. The invariant interval $\mathrm{ds}^{2}=\mathrm{c}^{2} \mathrm{t}_{0}{ }^{2}=\mathrm{c}^{2} \mathrm{dt}^{2}-\mathrm{dx}^{2}$, takes then the more general local form $\mathrm{ds}^{2}=\mathrm{g}_{\mathrm{ij}} \mathrm{dx}^{\mathrm{i}} \mathrm{dx}^{\mathrm{j}}$, introducing the formalism of general relativity, and leading to all its developments and consequences.

Since the variations of all quantities are very slight in almost standing waves, as compared with their standing waves constant values, the Lorentz transformation remains locally verified for standing waves, when the velocity of propagation of the field $\mathrm{C}(\mathrm{x}, \mathrm{t})$ reduces to $\mathrm{c}$ at first order approximation. Inversely, dynamical properties of almost standing waves arise from those of standing waves, through variation of constants method for velocities $\mathrm{c}$ and $\mathrm{v}$, while the mass density $\mu=\mathrm{u}^{2} / \mathrm{c}^{2}$ of the standing wave limit remains unaffected in first approximation, according to (13), in the continuity equation (16) written in the form (20)

$$
\begin{array}{cc}
\mathrm{d}(\mu \mathbf{V}) / \mathrm{dt}=-\nabla \mu \mathrm{C}^{2}+\nabla(\mu \mathrm{V})^{2} / 2 \mu & \mu \mathrm{d} \mathbf{V} / \mathrm{dt}=-\mu \nabla\left(\mathrm{C}^{2}-\mathrm{V}^{2} / 2\right) \\
\mathrm{d} \mathbf{V} / \mathrm{dt}=-\nabla\left(\mathrm{C}(\mathrm{x}, \mathrm{t})^{2}-\mathrm{V}(\mathrm{x}, \mathrm{t})^{2} / 2\right) & \mathrm{d} \mathbf{V} / \mathrm{dt}_{0}=-\nabla_{0} \mathrm{C}^{2}\left(\mathrm{x}_{0}, \mathrm{t}_{0}\right)=-\nabla_{0} \Phi\left(\mathrm{x}_{0}, \mathrm{t}_{0}\right) .
\end{array}
$$

The acceleration of an almost standing wave, either at rest or in motion, is locally independent of its energy density, equivalent to mass density of matter. This characterizes gravitation interaction (Elbaz, 2015). The gravitational potential $\Phi(\mathrm{x}, \mathrm{t})=\mathrm{C}^{2}(\mathrm{x}, \mathrm{t})$ is formally identical with the square of the local velocity of propagation of the field.

The relations (26) are consistent with physical origin of gravitation, exposed in 1912 by Einstein, in a preliminary article on general relativity of 1915, on Light velocity and static gravitation field, (Einstein,1912) He established the equation $\Delta \mathrm{c}=0$ in vacuum, generalizing the Poisson equation $\Delta \Phi=0$ for the gravitation potential.

\section{Application to Standard Model}

Following Einstein's program, the basic scalar field propagating at speed of light, allows to offer elements to complete the Standard Model. It shows that its incompleteness, regarding gravitation and size of particles, results from implicit approximations: from General Relativity framework with varying speed of light, to special relativity framework with constant value of light velocity in vacuum, and from extended field to point-like elementary particles, as geometrical optics approximations with very high frequencies undetectable and then hidden .

More generally, the basic scalar c-field brings theoretical economies by deriving formally and physically different laws or principles, like continuity and least action laws, otherwise admitted as fundamental in classical physics, and in Standard Model.

\subsection{Relativistic Foundations of Standard Model}

At the foundation of Quantum Mechanics, in non relativist framework, a single material particle like an electron was admitted as staying permanent in order to fit with experiment. Then in the non relativist Schrödinger's equation, or in its mathematical equivalent, the constant proper energy $\mathrm{m}_{0} \mathrm{c}^{2}$ is eluded or hidden. The lower frequencies of interactions yield remaining wave properties expressed by space partial differential equations, from which the position of the particle is determined. It led to specific and unusual properties of quantum mechanics, like superposition principle and uncertainty principle, and to the observer's statute, associated with the admitted experimental collapse of the extended space function toward a point-like position. 
Nevertheless, even in the non relativistic Schrödinger's equation, the fundamental rôle of the speed of light in vacuum, appeared already in the beginning of Quantum Mechanics. It was implicit since the Schrödinger's equation derived from the relativist Klein-Gordon's equation, as an approximation. Afterwards Dirac made it more explicit with his relativist spinorial equation.

Nowadays, the more general relativist Quantum Field Theory has introduced some distance with Quantum Mechanics description. For instance, a single quantum particle is no longer considered alone as necessarily permanent and isolated. It can be created or annihilated. Its mass is not a time-independent constant, but varies according to the Feynman process. Its experimental point-like behavior is not of prime importance, since it derives as a kind of resonance from a continuous specific field expressed by partial differential equations (Quigg, 1983).

The Einstein's program introduces a space-like $u$ amplitude function, in addition to the time-like $\psi$ function of quantum theory. The $\mathrm{u}$ amplitude function describes the space extension of a standing field behaving as a particle of matter. It introduces the energy density $u^{2}$, either when at rest or in motion, linked to the continuity equation (15) with the energy-momentum conservation law. Its central extremum allows to define the position of a particle as a dynamical variable (6), either $\mathrm{x}_{0}$ at rest or $\mathrm{x}$ in motion, with $\mathrm{x}_{0} \rightarrow \mathrm{x}$-vt, yielding the Lorentz transformation. In geometrical optics experimental approximation, for very small and undetectable wavelenghts tending towards zero (6), the particle behaves as point-like, and $\mathrm{u}\left(\mathrm{k}_{0} \mathrm{r}_{0}\right)$ reduces to a Dirac's distribution $\delta\left(\mathrm{r}_{0}\right)$.The $\mathrm{u}$ amplitude function variations yield interactions and the least action law (16) with the determination of the Lagrangian (17). These properties apply both in classical and in quantum domains.

The scalar field $\varepsilon$ according to the Einstein's program introduces the u amplitude function, with its important theoretical role at the base of interactions and energy laws, otherwise admitted as fundamental or as principles. Such a basic role of the $\mathrm{u}$ amplitude function, reduced to a point in the point-like particles approximation, is usually only formally implicit, or hidden.

\subsection{Quantum Foundations of Standard Model}

In the quantum domain, the scalar field $\varepsilon\left(\mathrm{r}_{0}, \mathrm{t}_{0}\right)$ of the Einstein's program brings some elements for the solution of the physical origin of the Planck's constant.

Such a question challenged Planck himself since the beginning.In a memory read in front of the Physical Society, he told how he devoted a long time about it: " since the day I formulated the law, I begun myself to attack to the problem of its true physical significance....my vain attempts to adjust the quantum of action to the framework of classical physics pursued many years, and costed me a lot of efforts..."

Since its introduction in 1900, the properties associated with the Planck's constant has evolved, following different discoveries in classical and in quantum physics domains.

In the beginning, the Planck's constant arised as a link between energies of oscillations of electrons and of light radiations, and then in exchange of energy between localized matter and extended wave light. In 1900, it was established in a classical dual framework, before the advent of special relativity. Electrons, as point-like particles with constant mass, were physically and geometrically heterogeneous with regard to extended electromagnetic waves. Nevertheless, in order to reason in a homogeneous field framework, Planck assimilated electrons to field oscillators.An important advance in quantum domain happended in the 1920 years, when Planck's constant application was extended from energy of exchanged radiation $\mathrm{E}=\mathrm{h} v$,to mass-energy of electron itself $\mathrm{E}=\mathrm{h} v=\mathrm{mc}^{2}$, giving rise to the second quantification process. But this generalization, for matter and interactions, increased the difficulty about its physical significance. It enforced its admitted fundamental character, leading to justify its founding role upon all particles in the Standard Model (Landau, 1962).

The Standard Model leans then upon two pillars,with two fundamental constants: classical special relativist with the speed of light $\mathrm{c}$, and quantum probabilist with the Planck's constant $\mathrm{h}$.

The speed of light properties apply in the quantum framework, while the reverse is not true. For instance, the above generalization was based upon the relativist mass-energy equivalence. On the other hand, only a part of formal properties of the classical invariant adiabatic process has been used in the quantum domain. Another part of its physical properties was carried out in 1911 by Ehrenfest, when he established that the Planck's constant was an adiabatic invariant. With such an introduced term, he defined a quantity which remained constant during energy transformations inside a cavity without outside exchanges. (Navarro, 2006) (Ehrenfest, 1911).

The Einstein's program deepens our theoretical understanding of the Planck's constant. It offers a homogeneous physical and geometrical field framework for energy of material particles and for electromagnetic interaction. The particles derive in the geometrical optics approximation for very high frequencies, undetectable experimentally. Through the almost standing solution of a c-field, it allows to retrieve the formal invariant adiabatic character of the Planck's 
constant, together with the simultaneous advent of the second quantification for matter. By comparison, physicists had to wait more than two decades to discover the second quantification.

However, despite such elements for the solution of the physical origin of the Planck's constant, the question of determining the hidden very high value of the frequency of material particles, remains unsolved. It leads particles to manifest experimentally as point-like, and for the corresponding invariant adiabatic constant to behave as a fundamental constant of general application.

\subsection{Size of Particles}

In the Standard Model, elementary particles such as electrons or quarks, have no known internal structure. They are considered as point-like. This is obviously an approximation, or an incomplete description, since their energy density should be infinite.This feature is consistent with the special relativity framework, in which a particle is necessarily a singularity of the field (Landau, 1962). Since the difference between space-like and time-like characters is absolute, the time-like function $\psi$ of quantum theory describes only the time evolution of a particle, but not its space extension. Consequently a quantum particle can be either point-like or extended. In this perspective, the size of fundamental particles is not of prime importance in Standard Model. The point-like character is implicitly admitted because of the Planck's constant in the Einstein's relation E=hv for massless particles like photons and gluons, and in the de Broglie's relation $\mathrm{E}=\mathrm{h} v=\mathrm{mc}^{2}$ for massive particles like electrons and quarks. All particles are admitted to derive from different specific extended fields expressed by partial derivative equations, and characterized by their different mass and their different geometrical symmetry (Quigg, 1983). From a physical point of view, it is obvious that a quantum particle cannot behave simultaneously as point-like and as extended field.

This is illustrated by the double-slit experiment, which plays an emblematic rôle in non relativist quantum mechanics. (Elbaz, 2015). An individual particle, like an electron or a photon, behaves as a point when it is directly, and closely, in contact with matter, at emission and detection, and when passing through material slits. Between these halting-places, it travels like a wave.

The relativist quantum theory is appropriate for extended fields, while experiment exhibits the point-like character. Massive particles derive as resonances of corresponding quantum fields, while massless particles manifest their point-like character during interactions with massive particles.

The Einstein's program allows to carry out a step toward harmonisation of field and particle in one hand, and toward unification of different specific fields in another hand.

It derives all particles from a basic scalar c-field, linked to the common $\mathrm{u}^{2}$ energy density, either self or exchanged, instead of specific different fields linked to each one. Massive particles, "as the regions in space where the field is extremely strong", correspond to standing fields. Theirspace-like bunched amplitude function $\mathrm{u}_{0}\left(\mathrm{k}_{0} \mathrm{r}_{0}\right)$, yield their point-like experimental behavior which appears only as an approximation for very high frequencies, tending towards a Dirac's distributionu $\mathrm{u}_{0}\left(\mathrm{k}_{0} \mathrm{r}_{0}\right) \rightarrow \delta\left(\mathrm{r}_{0}\right)$.

\subsection{Gravitation and Standard Model}

The problem of conciliating gravitation with the three other known interactions is probably the most important mark of incompleteness of the Standard Model. It has challenged numerous physicists since more than half a century. In general, they aim to quantize gravitation in order to incorporate it with the three others interactions. In one sense, they privilege the Planck's constant with regard to the speed of light, or the way for research, going from the local quantum microscopic to the general classical macroscopic.

By comparison, the experimental detection of gravitational waves, without gravitons as expected mediating quantum particles, followed the opposite way. It showed an increasing accuracy of Einstein's classical equations of General Relativity, and enforced some reliance upon determinism in physics.

From the c-field introduced by Einstein's program, two appreciable consequences arise: the formal identity of Planck's constant with an adiabatic invariant, and the difference of nature between gravitation due to speed of light variations, and electromagnetic interaction due to frequency variations.

In that perspective, the Einstein's program enlightens why the discovery of the quantum process was very closely linked with electromagnetism in black body light radiation, since adiabatic invariance and electromagnetism are due to frequency variations of the c-field, and why gravitation has until now resisted to its quantization, because of difference of physical nature between a speed and a frequency.

\section{Conclusion}

The Standard Model of Particles forms a quantum probabilistic consistent system, which gathers almost the whole present knowledge in theoretical physics. It describes the universe as constituted fundamentally of particles, both for 
matter and for interactions. It is incomplete since it does not include gravitation with the three other interactions, and leans upon elementary particles such as electrons or quarks, which are considered as point-like with no known internal structure.

These deficiencies, because of their very low physical actions, do not affect the high precision of experimental results. Nevertheless they point out basic approximations of the theory.

The recent experimental detection of gravitational waves, one century after their prediction by equations of General Relativity, in a classical determinist system, renewed scientific visibility of Einstein at the front of research. It occured after a long lapse of time, beyond and despite the prodigious successful development of the probabilistic approach of the quantum theory, to which he remained constantly, and resolutely opposed. He argued that the statistical behavior of quantum particles was due to incompleteness of equations. Einstein's successive discoveries convinced him that the core of the problem resided in the particle of matter, considered as a singularity, and then as a point-like approximation of a more fundamental field.

Consequently the Einstein's program is founded upon a scalar field $\varepsilon\left(\mathrm{r}_{0}, \mathrm{t}_{0}\right)$ propagating at speed of light $\mathrm{c}$. It offers not only means to circumvent the above difficulties, but, more generally, it represents a step toward unification and theoretical economy of physics. It forms a consistent classical determinist system for universe description.Standing waves correspond to matter, which kinematical and dynamical properties are formally identical. Their space-like amplitude function $\mathrm{u}_{0}\left(\mathrm{k}_{0} \mathrm{r}_{0}\right)$, supplements usual fundamental time-like equations of classical and quantum mechanics. Massive particles derive from the amplitude function in geometrical optics approximation conditions, when frequencies are infinitely high, and then hidden, yielding Dirac's distribution $\delta\left(\mathrm{r}_{0}\right)$, which describes a particle as a singularity. The scalar field $\varepsilon\left(\mathrm{r}_{0}, \mathrm{t}_{0}\right)$ brings theoretical economy by yielding also the energy-momentum conservation laws, and the least action law, with determination of the Lagrangian. The frequency variations of the field $\delta \Omega$ are linked to electromagnetic interaction, constituted by progressive waves. Quantum domain corresponds to wave optics approximation conditions, with wave-particle duality. Adiabatic variations of frequencies, give rise to introduction of an adiabatic constant, formally identical with Planck's constant, with unification of first quantification for interactions and second quantification for matter. Local variations of speed of light yield gravitation interaction and General Relativity framework.

The Einstein's program supplements, without affecting directly, the Standard Model of Particles, and the General Relativity for Gravitation. It offers a common framework for both. The scalar field $\varepsilon$ introduces a space-like $u$ amplitude function, exhibiting its important theoretical role for interactions and energy laws, otherwise admitted as fundamental or as principles. Such a basic role of the u amplitude function, is usually formally implicit, or hidden, when it is reduced to a point in the point-like particle geometrical optics approximation for very high frequencies or energies.

\section{References}

Dalibard, J. (2016). «Atomes et lumière en équilibre thermique: de l'argument d'Einstein aux mélasses optiques» College de France, Paris

Dimarcq, N. (2013). La mesure $d u$ temps, Institut Astrophysique Paris http://www.planetastronomy.com/special/2014-special/05nov/Dimarcq-IAP.htm

Ehrenfest, P. (1911). Welche züge der lichquantenhypothese spielen in der theorie der Wärmestrahlung eine wesentliche rolle?Ann. Phys., 35, 91-118.https://doi.org/10.1002/andp.19113411106

Einstein, A. (1912). Lichtgeswindigkeit und Statik des Gravitationsfeldes, Annalen der physik,4(38), 355-369.

Einstein, A. (1916). «Strahlungs-emission und absorption nach der quantentheorie », in Deutsche Physikalische Gesellschaft, 18, 318-323.

Einstein, A. (1920). The Aether and Relativity Theory. Leyde University, Leyde.

Einstein, A. (1929). Einstein's Theory of Relativity, conference

Einstein, A. (1936). Physics and reality, Journalof Franklin, Institute. https://doi.org/10.1016/S0016-0032(36)91047-5

Einstein, A. (1949). Philosopher, Scientist. Cambridge University Press, London. 51, 81

Einstein, A., \& Infeld, L. (1938). The Evolution of Physics. Cambridge Univ Press, Cambridge, 228-232.[6]

Elbaz, C. (1984). L'onde stationnaire et la transformation de Lorentz, CR. Acad. Sc Paris, T., 298, Serie II, 13, 543-546.

Elbaz, C. (2010). Asymptotic Analysis,68, 77-88.

Elbaz, C. (2012). Discrete and Continuous Dynamical Systems, A.I.M.S, Series B, 17, 835-849.

Elbaz, C. (2013). Annales de la Fondation Louis de Broglie, 38, 195-217. 
Elbaz, C. (2014). Wave particle duality in Einstein-de Broglie programs.Journal of Modern Physics, 5, 2192-2199. https://doi.org/10.4236/jmp.2014.518213

Elbaz, C. (2015). Gravitation and Electromagnetism Conciliated Following Einstein's Program. Journal of Modern Physics, 6, 660-669. https://doi.org/10.4236/jmp.2015.65072

Elbaz, C. (2015). On Einstein's Program and Quantum Mechanics,Applied Physics Research, 7(6), 126. ISSN 1916-9639, E-ISSN 1916-9647, Canadian Center of Science and Education.https://doi.org/10.5539/apr.v7n6p126

Elbaz, C. (2015). On Einstein"s Program and Quantum Double Slit Experiment,Studies in Engineering and Technology, 3(1). https://doi.org/10.11114/set.v3i1.1730

Landau, L., \& Lifchitz, E. (1960). Mechanics, Pergamon.

Landau, L., \& Lifchitz, E. (1962). The Classical theory of fields, Pergamon. P 102

Navarro, L., \& Perez, E. (2006). Paul Ehrenfest: The Genesis of the Adiabatic Hypothesis, 1911-1914, Arch. Hist. Exact. Sci., 60, 209-267. https://doi.org/10.1007/s00407-005-0105-1

Quigg, C. (1983). Gauge theories of the strong, weak, and electromagnetic interactions, Benjamin, Cummings Publishing Company,41.

Wikipedia, (2017). CERN, Standard Model of Elementary Particles. https://en.wikipedia.org/wiki/Elementary_particle

\section{Copyrights}

Copyright for this article is retained by the author(s), with first publication rights granted to the journal.

This is an open-access article distributed under the terms and conditions of the Creative Commons Attribution license which permits unrestricted use, distribution, and reproduction in any medium, provided the original work is properly cited. 